\title{
Considerações acerca das condições de vida de idosos no Vale do Jequitinhonha, Minas Gerais - Brasil
}

\author{
Considerations about life conditions of elderly in Jequitinhonha Valley, Minas Gerais - \\ Brazil \\ Saulo Sacramento Meira* \\ Alba Benemérita Alves Vilela** \\ Cezar Augusto Casotti**** \\ Ana Carolina França dos Anjos \\ Doane Martins da Silva ${ }^{* * * * *}$
}

\begin{abstract}
Resumo:
Teve-se como objetivo descrever as variáveis sociodemográficas, econômicas e das condições de saúde de idosos corresidentes em Salto da Divisa, Minas Gerais. Tratase de um estudo epidemiológico, de delineamento transversal, tipo inquérito domiciliar de base populacional, realizado com 279 idosos residentes na zona urbana e rural com idade maior ou igual a 60 anos, cadastrados nas (3) três Estratégias de Saúde da Família (ESF) que compõe o município e que não apresentavam déficit cognitivo segundo o Mini-Exame do Estado Mental (MEEM). Os dados foram obtidos por meio do questionário Brazil Old Age Schedule (BOAS) e analisados no SPSS ${ }^{\circledR}$ 15.0. Como principais achados, houve predominância do sexo feminino $(58,8 \%)$, sem escolaridade $(64,2 \%)$, com renda de até $1 \mathrm{SM}(81,7 \%)$, tenderam a coabitar mais com os netos $(71,0 \%)$, estavam satisfeitos com os coabitantes $(89,6 \%)$, possuíram autopercepção de saúde boa $(60,6 \%)$, consideraram-se felizes com a vida $(92,8 \%)$ e com boas expectativas para o futuro (52,0\%). O estudo revelou que apesar dos baixos determinantes sociais, os idosos em estado de corresidência no município possuíram bons aspectos psicossociais e sentimentos positivos mediante a vida, sugerindo que a configuração do ambiente familiar tem sido satisfatória para os participantes de Salto da Divisa.
\end{abstract}

Palavras-chave: Idoso; Habitação; Condições sociais; Envelhecimento.

\footnotetext{
* Fisioterapeuta, Mestre e Doutorando pelo Programa de Pós-Graduação de Enfermagem e Saúde da Universidade Estadual do Sudoeste da Bahia (PPGES/UESB). E-mail: saulo_meira@hotmail.com

** Enfermeira, Docente do Programa de Pós-Graduação de Enfermagem e Saúde da Universidade Estadual do Sudoeste da Bahia (PPGES/UESB). E-mail: alba_vilela@hotmail.com

*** Professor do Curso de Odontologia e do Programa de Pós-graduação em Enfermagem e Saúde da Universidade Estadual do Sudoeste da Bahia (UESB). Doutor em Odontologia Preventiva e Social. E-mail: cacasotti@uesb.edu.br.

**** Fisioterapeuta pela Universidade Estadual do Sudoeste da Bahia - UESB. E-mail: doane.ef@hotmail.com.

***** Enfermeira, Doutoranda pela escola de Enfermagem da Universidade Federal de Minas Gerais (UFMG). Email: doane.ef@hotmail.com.
} 


\begin{abstract}
:
The objective of this work is to describe the demographic, economic and health conditions variables of elderly co-residents in the municipality of Minas Gerais, since the longest life expectancy has awakened to the necessity of characterization of elderly populations. This is a cross-sectional epidemiological study, type population-based survey conducted with 279 elderly through a systematic sampling without replacement with sampling interval of 2, among those living in urban and rural areas aged greater than or equal to 60 years, enrolled in three (3) Family Health Strategies (ESF) that compose the municipality of Salto da Divisa and who had no cognitive impairment measured by the Mini Exam Mental State Examination (MMSE). Data were collected through the questionnaire Brazil Old Age Schedule (BOAS), tabulated in Epilnfo ${ }^{\circledR}$ 7.7.0.6 and analyzed using SPSS ${ }^{\circledR}$ 15.0. As main findings there were predominantly female (58.8\%), with no schooling (64.2\%), with income up to $1 \mathrm{SM}$ (81.7\%), tended to cohabit more with grandchildren (71.0\%), were satisfied with the cohabiting (89.6\%), possessed self-rated good health (60.6\%) considered themselves to be happy with life (92.8\%) and good expectations for the future (52,0\%). The study revealed that despite low social determinants, the elderly in a state of co residence in the municipality possessed good psychosocial aspects and positive feelings about life, suggesting that the configuration of the family environment has been satisfying for the participants.
\end{abstract}

Keywords: Aged; Housing; Social conditions; Aging.

\title{
Introdução
}

O envelhecimento populacional atualmente é uma característica observada tanto em países desenvolvidos quanto em desenvolvimento. No Brasil, esse processo esta acontecendo de forma bastante acelerada. O número de idosos no país passou de 3 milhões, em 1960, para 7 milhões, em 1975, atingindo 20 milhões em 2008. De acordo com projeções conservadoras, em 2020 o Brasil possuirá um contingente superior a 30 milhões de pessoas idosas (CARVALHO; GARCIA, 2003; VERAS, 2009).

A atenção às pessoas idosas deve estar voltada para as várias dimensões de sua vida, levando em consideração os aspectos familiares, econômicos e sociais desses indivíduos (DOMINGUES et al., 2013). Nesse contexto, a velocidade da expectativa de vida proporcionou também um aumento nas preocupações, bem como nas iniciativas governamentais em atender às demandas do idoso, principalmente em relação às ações direcionadas ao suporte social (GARCIA; RODRIGUES; BOREGA, 2002).

Assegurar prioritariamente o suporte a esses indivíduos é obrigação da família, da comunidade e do Estado, promovendo a efetivação do direito à vida, das condições de 
saúde, alimentação, educação de qualidade, ao lazer, ao trabalho, à cidadania, à liberdade, à dignidade, ao respeito e à convivência familiar e comunitária (BRASIL, 2003).

Com o crescimento da população idosa, emerge a necessidade de modificações estruturais nas políticas de serviços sociais, de saúde e a adequação dos valores culturais (LIMA-COSTA; BARRETO, 2003). Entretanto, os gastos elevados com assistência à saúde, a desigual distribuição de renda, as condições precárias de alimentação, escolaridade e habitação, determinantes sociais comuns em nosso país, geram expressivas repercussões sociais que afetam as condições de vida da população, em especial das pessoas acima de 60 anos.

O envelhecimento populacional, associado às modificações econômicas e sociais que acompanham a industrialização e a modernização das sociedades, tem afetado a constituição das famílias, independente do contexto cultural. A estrutura familiar esta em constante transformação e modificou-se, principalmente, em relação à composição, ao tamanho, aos papéis sociais tradicionais e a algumas funções familiares. Nesse contexto de mudanças no entorno familiar decorrentes do processo de envelhecimento populacional, destaca-se a conformação de um novo arranjo familiar, a corresidência (CAMARANO; EL GHAOURI, 2003).

Assim, a maior longevidade permite às pessoas viverem mais e amplia o número de famílias com a presença de idosos. Não raro uma família apresenta três ou quatro gerações que convivem entre si. No Brasil, o tamanho médio das famílias apresentou redução nas últimas décadas e, apesar da tendência decrescente, o tamanho médio de uma família de idoso, em 2000, era de 3,3 pessoas (CAMARANO et al., 2004).

A corresidência tem sido uma prática generalizada nos arranjos familiares com a presença de idosos, independente de o idoso ser ou não o chefe da família. Esta prática pode ocorrer devido ao adiamento da saída dos filhos por questões econômicas, como também pela inserção do idoso na residência dos filhos, decorrente da necessidade de cuidados, o que varia de acordo com o contexto cultural (DOMINGUES et al., 2013; CAMARANO et al., 2004).

Assim, é necessário o reconhecimento das variáveis sociodemográficas, econômicas e das condições de saúde de idosos que corresidem com familiares, com o propósito de oferecer subsídios para o planejamento local de ações de saúde, considerando a 
importância de se inserir este novo arranjo familiar como contexto de cuidados no Sistema Único de Saúde (SUS).

Portanto, neste estudo, definiu-se como objetivo descrever as variáveis sociodemográficas, econômicas e das condições de saúde de idosos corresidentes em Salto da Divisa, situados no vale do Jequitinhonha no Estado de Minas Gerais (MG), Brasil.

\section{Métodos}

\section{Tipo de Estudo e Sujeitos}

Trata-se de um estudo epidemiológico, com delineamento transversal, do tipo inquérito domiciliar de base populacional, realizado com indivíduos de idade maior ou igual a 60 anos, residentes na zona urbana e na zona rural do município de Salto da Divisa-MG.

Neste estudo, adotou-se como idoso corresidente aquele que compartilhava o domicílio com mais de uma pessoa. Foram pesquisados idosos em domicílios adstritos às (3) três Estratégias de Saúde da Família (ESF) existentes em Salto da Divisa, município de pequeno porte com população em torno de 6.858 habitantes (IBGE, 2010).

\section{Procedimentos de Coleta}

Inicialmente foi solicitado à Secretaria Municipal de Saúde de Salto da Divisa-MG que realizasse uma busca dos indivíduos acima de 60 anos de idade por meio do Sistema de Informação da Atenção Básica (SIAB) em cada uma das (3) três áreas adstritas, existentes no município. Em seguida, visitaram-se as ESF do município e junto com os Agentes Comunitários de Saúde (ACS) foram identificados e listados, por meio das "Fichas A", os idosos do município que viviam em estado de corresidência.

Após estimar o total de pessoas idosas que residiam em corresidência ( $n=699$ ), realizou-se o cálculo do tamanho amostral adotando-se os seguintes parâmetros: proporção de 50\% para o cálculo de populações finitas segundo Martins (2005), nível de confiança de $95 \%$ e erro de $5 \%$. 


\section{Amostra}

A amostra mínima representativa dessa população estabelecida para este estudo foi de 249 idosos à qual se acrescentaram 20\% prevendo-se as perdas, chegando-se ao valor representativo de 299 indivíduos. Em seguida, calculou-se o intervalo amostral estabelecendo-o em aproximadamente dois $(n=2)$. Concomitantemente, realizou-se o sorteio, para determinação dos participantes da pesquisa, obtendo-se aleatoriamente uma amostra a ser composta por 349 idosos. Ao final da obtenção dos dados da pesquisa, constatou-se que 70 idosos foram excluídos da amostra, 58 por não alcançarem a pontuação mínima do Mini-Exame do Estado Mental (MEEM) e 12 por recusas em participar da pesquisa, totalizando, então, 279 participantes.

Como critério de inclusão, estabelecido para a constituição da amostra desse estudo, os idosos deveriam estar cadastrados nas ESF, coabitar com uma ou mais pessoas e apresentar condições cognitivas que lhes permitissem responder aos questionamentos conforme o que estava proposto no MEEM.

Foram adotados como critérios para a supressão da participação do idoso neste estudo: não responder na íntegra os questionários; recusar participar da pesquisa; apresentar resultado inferior aos pontos de corte no MEEM, considerando o respectivo nível de escolaridade do idoso entrevistado e não se encontrar no domicílio após três (3) visitas efetuadas em dias e horários distintos.

\section{Instrumentos}

Para avaliação e rastreio de demências foi utilizado o MEEM, instrumento que fornece informações sobre diferentes parâmetros cognitivos, contendo questões agrupadas em sete categorias, cada uma delas planejada com o objetivo de avaliar "funções" cognitivas específicas, tais como a orientação temporal; orientação espacial; registro de três palavras; atenção e cálculo; recordação das três palavras; linguagem e capacidade construtiva visual. O escore do MEEM pode variar de um mínimo de 0 pontos, o qual indica o maior grau de comprometimento cognitivo dos indivíduos, ate um total máximo de 30 pontos, o qual, por sua vez, corresponde a melhor capacidade cognitiva (THAL; GRUNDMAN; GOLDEN, 1986; UHLMANN; LARSON; BUCHNER, 1987). 
O nível de corte foi estabelecido de acordo com a escolaridade (anos de estudo) declarada pelo idoso, diferencia-se entre: analfabetos (19 pontos); 1 a 3 anos de escolaridade ( 23 pontos); 4 a 7 anos ( 24 pontos) e maior que 7 anos de escolaridade (28 pontos).

Outro instrumento utilizado foi o questionário semiestruturado Brazil Old Age Schedule (BOAS), um construto multidimensional traduzido e validado para o Brasil, constituído por nove sessões, sendo elas: Informações Gerais; Saúde Física; Utilização de Serviços Médicos e Dentários; Atividades da Vida Diária; Recursos Sociais; Recursos Econômicos; Saúde Mental; Necessidades e Problemas que afetam o entrevistado e condições que contextualizam a vida do idoso (VERAS; DUTRA, 2008). Algumas questões do BOAS que envolviam itens e recursos com valores contemplados em outras sessões como saúde mental (informações já fornecidas pelo MEEM) e informações sobre estrutura física das residências, foram suprimidas a fim de reduzir o tempo de duração de entrevista (estratégia importante em pesquisa com idosos, uma vez que, no geral, tendem a apresentar baixa tolerância cognitiva), com a preocupação de não afetar a significância do questionário. Após a redefinição das estratégias, retornou-se ao campo de pesquisa para a obtenção dos dados da pesquisa.

Por se tratar de um instrumento multidimensional, o BOAS vem sendo cada vez mais empregado no Brasil por pesquisadores empenhados na temática do envelhecimento humano (BENEDETTI et al., 2008; BENEDETTI; MELLO; GONCALVES, 2007; PILGER; MENON; MATHIAS, 2011; VERAS; DUTRA, 2008).

As poucas opções de instrumentos de pesquisa voltados à investigação dos fatores multidimensionais que interagem junto à população idosa é um dos motivos que levaram à escolha desse instrumento. Além disso, é um inventário amplo e completo, analisando praticamente todas as interfaces com as quais o idoso se relaciona em termos sociocomunitários. Os estudos multidimensionais estão preenchendo cada vez mais uma lacuna que se estende há décadas, sustentada pelas pesquisas reducionistas, fragmentadas que não correspondem a um sentido de globalidade dos eventos, tratando de maneira inadequada a produção de saberes, realidade transversal e multidimensional (MORIN, 2013). 


\section{Análise dos dados}

Os dados produzidos por meio do questionário BOAS foram tabulados no Epilnfo ${ }^{\circledast}$ (versão 7.7.0.6) e analisados no SPSS ${ }^{\circledR}$ 15.0. Realizaram-se análises descritivas: média, mediana, mínimo, máximo, desvio-padrão, frequência simples e relativa.

\section{Aspectos Éticos}

A pesquisa foi aprovada por um Comitê de Ética em Pesquisa (CEP) sob o protocolo no 047/2009, como um subprojeto de uma pesquisa maior intitulada: "Envelhecimento e Corresidência: uma questão de gênero".

\section{Resultados}

De acordo com os dados pesquisados no município, houve maior proporção de idosos do sexo feminino (58,8\%), entre a faixa etária de 60 a 69 anos (52,0\%), média de idade de 70 anos (dp $\pm 7,3)$, variando entre 60 e 98 anos. Foi identificado percentual expressivo de analfabetos (64,2\%); entre os que possuíam escolaridade, a média foi de 1,8 anos $(\mathrm{dp} \pm 5,13)$.

No que diz respeito ao estado civil, $62 \%$ dos idosos eram casados ou viviam com o companheiro, possuindo em média $6,0(\mathrm{dp} \pm 4,05)$ filhos por domicílio. Referente aos aspectos da corresidência, observou-se que a média de indivíduos que coabitam com os idosos mineiros estudados foi de aproximadamente $2(\mathrm{dp} \pm 1,35)$ pessoas, sendo o neto (a) a geração mais frequente nos lares pesquisados, representando 71,0\% (tabela 1). Quanto à variável "satisfação com os coabitantes", 89,6\% dos idosos declararam estar satisfeitos.

Tabela 1 - Características sociodemográficas e econômicas, de pessoas idosas corresidentes em Salto da Divisa - Minas Gerais, 2013.

\begin{tabular}{|c|c|c|}
\hline Variável & $(n=279)$ & $\%$ \\
\hline \multicolumn{3}{|l|}{ Sexo } \\
\hline Feminino & 164 & 58,8 \\
\hline Masculino & 115 & 41,2 \\
\hline \multicolumn{3}{|l|}{ Faixa etária } \\
\hline $60-69$ anos & 145 & 52,0 \\
\hline 70-79 anos & 106 & 38,0 \\
\hline 80-89 anos & 28 & 10,0 \\
\hline \multicolumn{3}{|l|}{ Escolaridade } \\
\hline Sem Escolaridade & 179 & 64,2 \\
\hline
\end{tabular}




\begin{tabular}{lcc} 
Com Escolaridade & 100 & 35,8 \\
\hline Estado Conjugal & & \\
Casado/mora junto & 173 & 62,0 \\
Viúvo & 73 & 26,2 \\
Divorciado/Separado & 20 & 7,2 \\
Solteiro & 13 & 4,7 \\
\hline Ocupação & & \\
Lavrador & 96 & 34,4 \\
Serviços gerais & 31 & 11,1 \\
Dona de Casa & 30 & 10,8 \\
Outras Ocupações ${ }^{1}$ & 122 & 43,7 \\
\hline Renda Familiar & & \\
Até 1SM & 227 & 81,4 \\
Acima de 1SM & 52 & 18,6 \\
\hline O que ganha para necessidades básicas & & \\
Dá e sobra & 68 & 24,4 \\
Dá na conta & 97 & 34,8 \\
Sempre falta um pouco & 66 & 23,7 \\
Sempre falta muito & 48 & 17,2 \\
\hline Imóvel & & \\
Propriedade do idoso & 216 & 77,4 \\
Propriedade do cônjuge & 14 & 5,0 \\
Alugado & 13 & 4,7 \\
Cedida sem custo & 36 & 12,9 \\
\hline Situação econômica aos 50 anos & & \\
Melhor & 156 & 55,9 \\
A mesma & 53 & 19,0 \\
Pior & 70 & 25,1 \\
\hline Satisfação com corresidentes & & \\
Sim & 250 & \\
Não & 29 & \\
\hline 1 Outras profissões: Vaqueiro, costureira, lavadeira entre outras. & \\
2 SM = Salário Mínimo à época $=$ R\$678,00. & & \\
Fonte: Elaborada pelo autor. & & \\
& & \\
\hline & & \\
\hline
\end{tabular}

Com relação à renda mensal, 81,7\% declararam receber até 01 Salário Mínimo (SM), sendo a média da renda calculada em $\mathrm{R} \$ 790,00$ (valor mínimo de $\mathrm{R} \$ 678,00$ e máximo de $\mathrm{R} \$ 8.000)$. Para 23,7\% dos idosos, a situação econômica atual foi classificada como "melhor que aos 50 anos de idade", e 34,8\% declararam que a renda "dá na conta" para todas as despesas.

Quanto à autopercepção da saúde, 60,6\% consideram o seu estado de saúde "Bom", e, comparada à saúde de outros idosos nas mesmas condições de vida, $47,3 \%$ acreditam que o seu estado de saúde é melhor (tabela 2). Ainda quanto às questões de saúde, $84,2 \%$ referiram possuir alguma patologia, sendo a Hipertensão Arterial Sistêmica (HAS) a mais 
prevalente $(46,2 \%)$, seguida dos problemas osteomusculares $(24,0 \%)$. A presença de doenças foi considerada por $41,2 \%$ dos idosos como o principal motivo limitante para a realização das atividades diárias.

Quanto à percepção dos aspectos psicossociais, $71,3 \%$ dos idosos não relatam arrependimento diante a vida, $92,8 \%$ se declaram felizes e $52,0 \%$ revelam possuir boas expectativas de vida para o futuro.

Tabela 2 - Autopercepção de saúde de idosos corresidentes em Salto da Divisa - MG, 2013.

\begin{tabular}{lcc}
\hline Variável & (n=279) & \% \\
\hline Autopercepção da saúde & & \\
Ótima & 24 & 8,6 \\
Boa & 169 & 60,6 \\
Ruim & 64 & 22,9 \\
Péssima & 22 & 7,9 \\
\hline Sua saúde comparada à de idosos da mesma idade & & \\
Melhor & 132 & 47,3 \\
lgual & 122 & 43,7 \\
Pior & 25 & 9,0 \\
\hline Possui problemas de saúde & & \\
Sim & 235 & 84,2 \\
Não & 44 & 15,8 \\
\hline Morbidades autorreferidas & \multicolumn{3}{|c}{} \\
Problemas osteomusculares & 67 & 24,0 \\
Hipertensão arterial & 129 & 46,2 \\
Diabetes & 19 & 6,8 \\
Outras doenças & 64 & 23,0 \\
\hline Fonte: Dados da pesquisa. & \multicolumn{3}{l}{} \\
\hline
\end{tabular}

\section{Discussão}

De acordo resultados do IBGE (2012), e outros estudos de base populacional que revelam a maior composição da população idosa como sendo composta por mulheres, tal característica tem sido associada aos efeitos da mortalidade diferencial por sexo, evidenciando a feminização do envelhecimento humano (MEIRELES et al., 2007; LIMACOSTA; BARRETO; GIATTI, 2003; FREITAS et al., 2010).

As características ligadas ao sexo têm sido atribuídas à menor exposição das mulheres a determinados fatores de risco do que os homens, diferenças quanto à atitude 
em relação a doenças, incapacidades e pela maior cobertura da assistência ginecoobstétrica; ao mesmo tempo, as mulheres procuram mais os serviços de saúde, fazem mais uso de medicamentos e apresentam maiores taxas de adesão aos programas de prevenção (VERAS et al., 1988; KALACHE, 2007; PILGER; MENON; MATHIAS, 2009; IBGE, 2012; LIPOSCKI, 2007; GIACOMIN et al., 2008; LOYOLA FILHO; UCHOA; LIMA-COSTA, 2006; PILGER; MENON; MATHIAS, 2011).

Quanto à prevalência da faixa etária, esse resultado relaciona-se ao padrão demográfico brasileiro atual. Dados semelhantes são descritos pelo IBGE (2012) que aponta para a mesma faixa etária um percentual de $42,4 \%$ demonstrando maior representatividade de "idosos jovens" nesse grupo populacional, em decorrência de uma transição demográfica relativamente recente, considerando-se o período de reforma sanitária a partir da década de 1970.

A perpetuação do analfabetismo entre a população de maior idade expõe o problema como uma grave e urgente questão política e social a ser enfrentada pelo Estado e pela sociedade brasileira. Atualmente o analfabetismo é considerado um dos indicadores mais precisos para identificar o nível socioeconômico de uma população. Durante as décadas de 1930 e 1950, o ensino fundamental era restrito a segmentos sociais específicos. Até a década de 1980, os baixos níveis de escolaridade não eram considerados como fator limitante do desenvolvimento, sendo aceitáveis pelo modelo econômico, industrial e tecnológico do Brasil. Sendo assim, os idosos que vivem na atual conjuntura social advindo de um contexto expressivamente rural não conviveram com a necessidade de um maior grau de instrução para se desenvolver social e economicamente (LIPOSCKI, 2007; CRUZ, 2009).

É importante destacarmos que a baixa escolaridade limita o usufruto de bens e produtos culturais, impedindo uma boa carreira profissional, reduz os salários e limita também a defesa dos próprios direitos, constituindo-se importante fator de exclusão social. Ainda nesse contexto, a ocupação de lavrador foi a atividade remunerada mais desenvolvida pelos idosos entrevistados, remetendo a uma organização social e econômica do começo do século passado, que limitava o acesso à escola e aos serviços de saúde aos mais pobres (OLIVEIRA, 2011; FELICIANO; MORAES; FREITAS 2004). 
Em relação ao estado conjugal, é importante considerar que, apesar de a maior parcela da população estudada ser constituída por mulheres, não houve diferença expressiva entre os sexos, apontando que o casal de idosos tem envelhecido juntos. Outro indicador que sustenta essa informação se refere à média de sobrevida em torno de 70 anos de idade, valor abaixo da média nacional (73 anos), equiparando, assim, a diferença de expectativa de vida entre homens e mulheres (IBGE, 2012).

Diante desse contexto domiciliar, estudos indicam que os indivíduos casados apresentam melhores condições de saúde e maior sobrevivência quando comparados aos solteiros, viúvos e divorciados. O casamento parece funcionar como um conjunto de mecanismos causais, constituído por fatores ambientais, sociais e psicológicos, o que faz do estado de casado mais saudável do que os demais (GRUNDY, 2001; NORGREN et al., 2004).

Quanto ao arranjo familiar, verificou-se que os idosos que corresidem no município convivem em média com 2 pessoas, índice expressivamente baixo se comparado aos dados do IBGE (2010), que classifica a média de habitantes por domicílio de 3 pessoas. Essa informação sugere uma característica local do município pesquisado, uma vez que as baixas condições de emprego e renda forçam os indivíduos mais jovens ao êxodo para outros centros urbanos, diminuindo a rede de apoio desses idosos que tendem a permanecer no domicilio.

Esse arranjo familiar em sua maioria composta por netos na corresidência se deve, em grande parte, ao fato de os idosos funcionarem como único apoio para os netos e filhos, seja para que esses trabalhem, por deficiência ou mesmo pelo insucesso na tentativa da vida fora do contexto familiar. Conviver conjuntamente tem ainda se mostrado uma solução para a solidão e para a falta de recursos econômicos entre os membros (SILVA; GALERA; MORENO, 2007; CRUZ, 2009).

Ainda nos aspectos que envolvem o arranjo familiar, a declaração de satisfação positiva entre os idosos com as demais pessoas que compartilham o domicilio tende a apresentar significativas relações, podendo estar baseada na "teoria das trocas", em que Sir. James Frazer, em 1919, ofereceu a explicação econômica para quando as pessoas não possuem propriedade de valor suficiente para trocar por outro bem, então elas dão algo de valor relativo (TURNER, 1996). 
Os pressupostos do processo de troca é o resultado de esforços dos indivíduos para preencher em necessidades básicas em que há produção de retornos para os integrantes, levando à padronização das interações sociais. Os padrões de interação não somente servem às necessidades individuais, mas também restringem os tipos de estruturas sociais que podem emergir e levam à diferenciação de poder e privilégio em grupos sociais. Outra explicação, de cunho social, é a de que a troca esta fundamentada na reciprocidade do cuidado entre os membros, que tende a gerar sentimentos de confiança e gratidão. Em ambas as situações a interação é mantida por ser considerada compensadora (TURNER, 1996).

Verificou-se que a aposentadoria é a fonte de renda que constitui importante recurso, ou mesmo a principal fonte de renda da família, e, conforme apontam Camarano et al., (2004), os benefícios da Seguridade Social nos domicílios onde há a presença dos idosos são de suma importância para a composição da renda dessa família. Entre os anos de 1997 e 2007, houve o incremento de 6\% de idosos que contribuem financeiramente com os lares, somando $53 \%$ dos domicílios brasileiros onde os idosos contribuíam com mais da metade da renda familiar. Nas regiões Sudeste e Sul as proporções dos domicílios com tal contribuição foram de 46,9\% e 52,2\%, respectivamente, sendo ainda maior nas regiões mais carentes do país (IBGE, 2008).

A renda mensal média dos idosos apresentou valores acima do esperado $(R \$ 790,00)$, diferentemente de outros estudos em que populações com características semelhantes apresentaram valores menores a de um salário mínimo, apontando para a alta concentração de renda na população estudada podendo estar relacionada à permanência do modelo agropecuário tradicional no município (TAVARES et al., 2011; ROSSET et al., 2011).

Apesar do recurso financeiro questionável quanto à cobertura das necessidades básicas, os benefícios previdenciários garantem ao idoso uma condição mínima de autonomia financeira. Neste contexto, é importante destacar o papel do Benefício de Amparo ao Idoso pago aos maiores de 65 anos que não têm condições de se manter financeiramente, mesmo não havendo contribuído para a Previdência Social durante a vida. Quanto às questões dos imóveis, $77.4 \%$ declararam ser os proprietários dos domicílios, sejam herdados dos cônjuges, sejam adquiridos mediante maior acesso ao 
crédito, reforçando a importância do papel do idoso para estrutura dos membros que coabitam.

Quanto às condições de saúde, detectou-se um expressivo percentual de idosos portadores de distúrbios patológicos. Para Gottlieb et al., (2011) o envelhecimento de modo geral esta associado a um novo perfil de morbimortalidade, caracterizado por uma maior probabilidade de acometimento por Doenças Crônicas Não Transmissíveis (DCNT). Além das alterações morfofisiológicas e funcionais que estão relacionadas com o avanço da idade, o estilo de vida, principalmente, o sedentarismo e a dieta desbalanceada, contribuem também para o aumento da prevalência de DCNT e mortalidade (CIOLAC; GUIMARÃES, 2004).

Quanto às morbidades autorreferidas de hipertensão arterial encontradas neste estudo, acompanha a elevada tendência descrita em outros estudos da mesma natureza como Porciúncula (2012) e Pilger, Menon, Mathias (2011), que encontraram percentuais de $59,0 \%$ e $34,9 \%$, respectivamente, nas populações estudadas. Esse dado preocupante alerta para a necessidade em intensificar as práticas desenvolvidas para o controle da HAS no município, ao considerarmos a forte interferência que essa doença referida pode impactar na qualidade de vida desses indivíduos.

Essa mesma preocupação serve para as doenças osteomusculares, na qual inclui a artrite e artrose. Segundo um estudo norte-americano, esse tipo de manifestação representa a principal causa de incapacidades na população idosa de seu país, e espera-se que sua prevalência aumente com o envelhecimento populacional o que altera a rotina desses idosos, repercutindo indiretamente nas limitações físicas, aumentando as demandas de cuidados e dependência por parte desses gerontes (HOOTMAN et al., 2006). Essas manifestações tendem a modificar a rotina familiar, o que pode impactar no bemestar do idoso e de todos os coabitantes.

Quanto à autopercepção de saúde, 60,6\% dos entrevistados consideraram seu estado "Bom", ao mesmo tempo que classificam sua saúde como "Melhor" se comparada a outras pessoas da mesma idade. Dados semelhantes foram encontrados por Feliciano; Moraes; Freitas (2004) com idosos em comunidade no oeste de Belo Horizonte, no qual $40 \%$ classificaram seu estado de saúde como "Bom". A concepção desse aspecto é um forte 
indicador do estado real ou objetivo de saúde das pessoas, podendo ser considerada uma representação das avaliações objetivas ligada ao bem-estar.

É importante considerar que o envelhecimento conduz a atitudes diferentes frente às limitações e incapacidades, podendo levar em alguns casos a adaptações diante das mudanças à medida que elas vão ocorrendo. Outra hipótese se refere a maior aceitação dessas alterações do estado de saúde, partindo do entendimento que as mesmas fazem parte de um envelhecer natural, de acordo com uma visão socialmente construída. Para Porciúncula (2012); Borges et al., (2008), os idosos associam a perda da capacidade funcional ao envelhecimento e não à perda da saúde, sendo comum afirmarem que possuem boa saúde para a idade quando perguntados.

Foi possível identificar ainda que a maioria dos idosos que vivem em estado de corresidência não relata arrependimentos com os fatos da vida. Eles tendem a se considerar felizes e possuem boas expectativas para o futuro. Esses dados indicam que os idosos que compartilham o domicílio com outras pessoas possuem condições de vida mais prazerosa e positiva, resultados semelhantes são encontrados em outros trabalhos descritos na literatura (RUIZ, 2007; MROCZEK; SPIRO, 2005). Essa repercussão positiva sugere que a corresidência no município tem sido interessante para o idoso no momento que aponta para boas relações no ambiente familiar entre os membros, o que sugere apoio e estímulo, fundamentais ao suporte emocional, financeiro e de segurança ante os desafios sociais em que estão contextualizados.

\section{Considerações finais}

Em relação às características sociodemográficas e econômica dos idosos corresidentes no município de Salto da Divisa - MG foi possível identificar que são, em sua maioria, "idosos jovens", do sexo feminino, sem escolaridade, lavradores, de baixa renda e apresentam como principais problemas de saúde a HAS e as doenças osteomusculares, as quais interferem em sua rotina de atividades diárias. Quanto às questões de moradia, os idosos dividem o domicílio com poucos habitantes, em grande parte com a presença de netos. Embora relatem boa percepção de saúde e satisfação com a vida, o município possui desafios na atenção à saúde das pessoas com mais de 60 anos de idade. 
A coleta de informações junto à população idosa na comunidade, investigando aspectos socioeconômicos e percepção de saúde individual, torna-se indispensável para gestores públicos, pois auxilia na implementação de estratégias e ações políticas que irão favorecer o bem-estar físico, mental e social dos idosos, baseadas não somente em seus direitos, mas em suas verdadeiras necessidades.

Acredita-se que conhecer o perfil sociodemográfico e de saúde de idosos corresidentes torna-se relevante, face a conformação desse novo arranjo familiar, decorrente do processo de envelhecimento populacional, com repercussões nos diversos âmbitos, aqui inclusa a família. Espera-se, então, que este estudo amplie o debate e suscite novos estudos sobre a conformação da família intergeracional, ancorada na corresidência, no contexto do envelhecimento populacional.

\section{Referências}

BENEDETTI, T. R. B. et al. Atividade física e estado de saúde mental de idosos. Revista de Saúde Pública, São Paulo, v. 42, n. 2, p. 302-307, abr. 2008. Disponível em: $<$ http://www.scielosp.org/scielo.php?script=sci_arttext\&pid=S0034$89102008000200016 \&$ Ing=en\&nrm=iso>. Acesso em: 20 jan. 2014.

BENEDETTI, T. B.; MELLO, A. L. S. F.; GONCALVES, L. H. T. Idosos de Florianópolis: autopercepção das condições de saúde bucal e utilização de serviços odontológicos. Ciência \& Saúde Coletiva, Rio de Janeiro, v. 12, n. 6, p. 1683-1690, Dec. 2007. Disponível em: <http://www.scielo.br/scielo.php?script=sci_arttext\&pid= S141381232007000600029\&Ing=en\&nrm=iso>. Acesso em: 20 jan. 2014

BORGES, J. M. M. et al. Perfil dos idosos frequentadores de grupos de convivência em Belo Horizonte, Minas Gerais, Brasil. Cadernos de Saúde Pública, Rio de Janeiro, v. 24, n. 12, p. 2798-2808, dez. 2008. Disponível em: <http://www.scielo.br/pdf/csp/v24n12/08.pdf>. Acesso em: 20 ago. 2013.

BRASIL. Lei no 10.741, de 01 de outubro de 2003. Dispõe sobre o Estatuto do Idoso e dá outras providências. Diário Oficial da União, Brasília, 3 out. 2003. Disponível: <http://bd.camara.gov.br/bd/bitstream/handle/bdcamara/763/estatuto_idoso_5ed.pdf>. Acesso em: 15 jul. 2013.

CAMARANO, A. A.; EL GHAOURI, S. K. Famílias com idosos: ninhos vazios? Texto para Discussão, Rio de Janeiro, n. 950, p. 1-21, abr. 2003. Disponível em URL: http://repositorio.ipea.gov.br/bitstream/11058/2879/1/TD_950.pdf>. Acesso em: 12 jan. 2012. 
CAMARANO, A. A. et al. Famílias: espaço de compartilhamento de recursos e vulnerabilidades. In: CAMARANO, A. M. (Org.). Os novos idosos Brasileiros: muito além dos 60? Rio de janeiro: IPEA, 2004. Cap. 5, p. 137-65.

CARVALHO, J. A. M.; GARCIA, R. A. O envelhecimento da população brasileira: um enfoque demográfico. Cadernos de Saúde Publica, Rio de Janeiro, v. 19, n. 3, p. 725-33, 2003. Disponível em: <http://www.scielo.br/pdf/csp/v19n3/15876.pdf>. Acesso em: 15 jul. 2013.

CIOLAC, E. G.; GUIMARÃES, G. V. Exercício físico e síndrome metabólica. Revista Brasileira de Medicina do Esporte, São Paulo, v. 10, n. 4, p. 319-24, 2004. Disponível em: <http://www.scielo.br/pdf/rbme/v10n4/22048.pdf>. Acesso em: 20 ago. 2013.

CRUZ, I. R. Avaliação geriátrica global dos idosos mais velhos residentes em Ribeirão Preto (SP) e Caixas do Sul (RS): indicadores para envelhecimento longevo. 2009. Tese (Doutorado) - Universidade de São Paulo, São Paulo, 2009. Disponível em: <www.teses.usp.br/teses/disponiveis/83/83131/tde-08012010-133459/pt-br.php>. Acesso em: 9 abr. 2013.

DOMINGUES, M. A. et al. Redes de relações sociais dos idosos residentes em Ermelino Matarazzo, São Paulo: um estudo epidemiológico. Revista Brasileira de Geriatria e Gerontologia, Rio de Janeiro, v. 16, n. 1, p. 49-59, 2013. Disponível em: <http://www.scielo.br/scielo.php?pid=S1809-98232013000100006\&script=sci_arttext>. Acesso em: 15 jul. 2013.

FELICIANO, A. B.; MORAES, S. A. de; FREITAS, I. C. M. de. O perfil do idoso de baixa renda no Município de São Carlos, São Paulo, Brasil: um estudo epidemiológico. Cadernos de Saúde Pública, Rio de Janeiro, v. 20, n. 6, p. 1575-1585, nov./dez. 2004. Disponível em: $<$ http://www.scielo.br/scielo.php?script=sci_arttext\&pid=S0102311X2004000600015\&Ing $=e n \& n r m=i s o>$. Acesso em: 8 maio 2013.

FREITAS, D. H. M. de et al. Autopercepção da saúde e desempenho cognitivo em idosos residentes na comunidade. Revista de Psiquiatria Clínica, São Paulo, v. 37, n. 1, p. 32-35, 2010. Disponível em: <http://www.scielo.br/scielo.php?script=sci_arttext\&pid=S010160832010000100007>. Acesso em: 9 abr. 2013.

GARCIA, M. A. A.; RODRIGUES, M. G.; BOREGA, R. dos S. O envelhecimento e a saúde. Revista de Ciências Médicas, Campinas, v. 11, n. 3, p. 221-231, set./dez. 2002.

GIACOMIN, K. et al. Estudo de base populacional dos fatores associados à incapacidade funcional entre idosos na Região Metropolitana de Belo Horizonte, Minas Gerais, Brasil. Cadernos de Saúde Pública, Rio de Janeiro, v. 24, n. 6, p. 1260-1270, jun. 2008. Disponível em: <http://www.scielo.br/pdf/csp/v24n6/07.pdf>. Acesso em: 20 ago. 2013.

GOTTLIEB, M. G. V. et. al. Envelhecimento e Longevidade no Rio Grande do Sul: um perfil histórico, étnico e de morbi-mortalidade dos idosos. Revista Brasileira de Geriatria e Gerontologia, Rio de Janeiro, v. 14, n. 2, p. 365-380, 2011. Disponível em: 
$<$ http://revista.unati.uerj.br/scielo.php?script=sci_arttext\&pid=S18099823201100020001 6\&lng=pt\&nrm=iso>. Acesso em: 15 jul. 2013.

GRUNDY, E. Living arrangements and the health of older persons in developed countries. 2001. Disponível em: <http://www.un.org/esa/population/publications/ bulletin42_43/grundy.pdf>. Acesso em: 20 ago. 2013.

HOOTMAN, J. et al. Prevalence of doctor-diagnosed arthritis and arthritis- attributable activity limitation - United States, 2003-2005. MMWR Morb Mortal Wkly Rep., Atlanta, v. 55, p. 1089-92, 2006. Disponível em: <http://www.cdc.gov/mmwr/pdf/wk/mm5939.pdf>. Acesso em: 20 ago. 2013.

IBGE. Projeção da população do Brasil por sexo e idade, 1980-2050: revisão 2008. Rio de Janeiro: IBGE, 2008.

Sinopse do Censo Demográfico 2010: Brasil. 2010. Disponível em: <http://www.censo2010.ibge.gov.br/sinopse/>. Aceso em: 12 jan. 2012.

Síntese de indicadores sociais: uma análise das condições de vida da população brasileira. Rio de Janeiro: IBGE, 2012. Disponível em:

<ftp://ftp.ibge.gov.br/Indicadores_Sociais/Sintese_de_Indicadores_Sociais_2012/SIS_201 2.pdf>. Acesso em: 15 jul. 2013.

KALACHE, A. Fórum envelhecimento populacional e as informações de saúde do PNAD: demandas e desafios contemporâneos. Cadernos de Saúde Pública, Rio de janeiro, v. 23, n. 10, p. 2503-5, out. 2007. Disponível em: <http://www.scielo.br/pdf/ csp/v23n10/24.pdf>. Acesso em: 8 maio 2013.

LIMA-COSTA, M.; BARRETO, S. M. Tipos de estudos epidemiológicos: conceitos básicos e aplicações na área do envelhecimento. Epidemiologia e Serviços de Saúde, Brasília, v. 12, n. 4, p. 189-201, 2003. Disponível em:

<http://scielo.iec.pa.gov.br/pdf/ess/v12n4/v12n4a03.pdf>. Acesso em: 15 jul. 2013.

LIMA-COSTA, M. F.; BARRETO, S. M.; GIATTI, L. Condições de saúde, capacidade funcional, uso de serviços de saúde e gastos com medicamentos da população idosa brasileira: um estudo descritivo baseado na Pesquisa Nacional por Amostra de Domicílios. Cadernos de Saúde Pública, Rio de Janeiro, v. 19, n. 3, p. 735-43, 2003. Disponível em: <http://www.scielosp.org/pdf/csp/v19n3/15877.pdf>. Acesso em: 20 ago. 2013.

LIPOSCKI, D. B. A influência de um programa de intervenção psicomotora na aptidão psicomotora de idosos longevos. 2007. Dissertação (Mestrado em Ciências do Movimento Humano) - Universidade do Estado de Santa Catarina, Florianópolis, 2007. Disponível em: $<$ http://www.tede.udesc.br/tde_busca/arquivo.php?codArquivo=1384>. Acesso em: 20 ago. 2013.

LOYOLA FILHO, A. de; UCHOA, E.; LIMA-COSTA, M. Estudo epidemiológico de base populacional sobre uso de medicamentos entre idosos na Região Metropolitana de Belo Horizonte, Minas Gerais, Brasil. Cadernos de Saúde Pública, Rio de Janeiro, v. 22, n. 12, p. 
2657-67, dez. 2006. Disponível em: <http://www.scielo.br/scielo.php? script=sci_arttext\&pid=S0102-311X2006001200015>. Acesso em: 20 ago. 2013.

MARTINS, G. A. Estatística geral e aplicada. 3. ed. São Paulo: Atlas, 2005.

MEIRELES, V. et al. Características dos idosos em área de abrangência do Programa Saúde da Família na região noroeste do Paraná: contribuições para a gestão do cuidado em enfermagem. Saúde e Sociedade, São Paulo, v. 16, n. 1, p. 69-80, jan./abr. 2007.

Disponível em: <http://www.scielo.br/scielo.php?pid=S010412902007000100007\&script=sci_arttext>. Acesso em: 15 jul. 2013.

MORIN, E. A cabeça bem feita: repensar a reforma, reformar o pensamento. 8. ed. Rio de Janeiro, 2003. Disponível em:

<http://www.uesb.br/labtece/artigos/A\%20Cabe\%C3\%A7a\%20Bem-feita.pdf>. Acesso em: 15 jan. 2014.

MROCZEK, D.; SPIRO, A. Change in life satisfaction during adulthood: findings from the veterans affairs normative aging study. Journal of Personality and Social Psychology, Washington, v. 88, n. 1, p. 189-202, Jan. 2005. Disponível em: <http://www.ncbi.nlm.nih.gov/pubmed/15631584>. Acesso em: 8 mai. 2013.

NORGREN, M. de B. P. et al. Satisfação conjugal em casamentos de longa duração: uma construção possível. Estudos de Psicologia, Natal, v. 9, n. 3, p. 555-584, Sep./Dec. 2004. Disponível em: <http://www.scielo.br/scielo.php?script=sci_arttext\&pid=s1413294×2004000300020>. Acesso em: 8 mai. 2013.

OLIVEIRA, M. Escolaridade dos idosos no Brasil é muito baixa. Brasília: Confederação Brasileira de Aposentados, Pensionistas e Idosos, 2011. Disponível: <http://www.cobap.org.br/capa/lenoticia.asp?ID=56306\#.T4I0U8gS_Ws.facebook>. Acesso em: 20 ago. 2011.

PILGER, C.; MENON, M. H.; MATHIAS, T. A. F. Características sócio-demográficas e de saúde de idosos dependentes residentes em domicílio. Revista Espaço para a Saúde, Londrina, v. 10, n. 2, p. 12-17, jun. 2009. Disponível em:

<http://www.uel.br/ccs/espacoparasaude/v10n2/Artigo3.pdf>. Acesso em: 20 ago. 2013.

PILGER, C. MENON, M. H.; MATHIAS; T. A. de F. Socio-demographic and health characteristics of elderly individuals: support for health services. Revista LatinoAmericana de Enfermagem, Ribeirão Preto, v. 19, n. 5, p. 1230-1238, set./out. 2011. Disponível em: <http://www.scielo.br/scielo.php?script=sci_arttext\&pid=S0104$11692011000500022>$. Acesso em: 20 ago. 2013.

PORCIÚNCULA, R. de C. R. da. Perfil socioepidemiológico dos idosos longevos em Recife, nordeste do Brasil. 2012. Dissertação (Mestrado Acadêmico em Saúde Pública) Fundação Oswaldo Cruz, Centro de Pesquisas Aggeu Magalhães, Recife, 2012. Disponível em: <http://www.cpqam.fiocruz.br/bibpdf/2012porciuncula-rcr.pdf>. Acesso em: 20 ago. 2013. 
ROSSET, I. et al. Diferenciais socioeconômicos e de saúde entre duas comunidades de idosos longevos. Revista de Saúde Pública, Rio de Janeiro, v. 45, n. 2, p. 3 91-400, 2011. Disponível em: <http://www.scielo.br/scielo.php?script=sci_arttext\&pid =S003489102011000200018\&lng=en\&nrm=iso>. Acesso em: 6 jul. 2013

RUIZ, T. Avaliação do grau de satisfação dos idosos com a qualidade de vida em um pequeno município do Estado de São Paulo. Revista APS, Juiz de Fora, v. 10, n. 1, p. 4-13, jan./jun. 2007. Disponível em: <http://www.ufjf.br/nates/files/2009/12/Avaliacao.pdf>. Acesso em: 15 jul. 2013.

SILVA, L.; GALERA, S. A. F.; MORENO, V. Encontrando-se em casa: uma proposta de atendimento domiciliar para famílias de idosos dependentes. Acta Paulista de Enfermagem, São Paulo, v. 20, n. 4, p. 17-25, 2007. Disponível em: <http://www.scielo.br/scielo.php?pid=S0103-21002007000400002\&script=sci_arttext>. Acesso em: 20 ago. 2013.

TAVARES, V. O. et al. Interfaces entre a renda dos idosos aposentados rurais e o contexto familiar. Textos \& Contextos, Porto Alegre, v. 10, n. 1, p. 94 -108, jan./jul. 2011. Disponível em: <http://revistaseletronicas.pucrs.br/ojs/index.php/fass/article/viewFile/8725/6427>. Acesso em: 2 set. 2013.

THAL, L. J.; GRUNDMAN, M.; GOLDEN, R. Alzheimer's disease: a correlational analysis of the blessed information - memory-concentration test and the mini-mental state exam. Neurology, Minneapolis, v. 36, p. 262-264, 1986.

TURNER, B. T. parsons on economic and social theory: The relevance of the amherst term papers. The American Sociologist, Sant Louis, v. 27, n. 4, p. 41-47, 1996. Disponível em: <http://link.springer.com/article/10.1007\%2FBF02692051>. Acesso em: 15 jul. 2013.

UHLMANN, R. F.; LARSON, E. B.; BUCHNER, D. M. Correlations of mini mental state and modified dementia rating scale to measures of transitional health status in dementia. Journal of Gerontology, Washington, v. 42, p. 33-36, 1987.

VERAS, R. P. et al. Research on elderly populations--the importance of the methods andtraining of the team: a methodologic contribution. Revista de Saúde Pública, São Paulo, v. 22, n. 6, 1988. Disponível em: <http://www.scielo.br/scielo.php?script=sci_ arttext\&pid=S0034-89101988000600008>. Acesso em: 12 abr. 2013.

VERAS, R. P. Envelhecimento, demandas, desafios e inovações. Revista de Saúde Pública, São Paulo, v. 43, n. 3, p. 548-54, 2009. Disponível em:

$<$ http://www.scielo.br/scielo.php?script=sci_arttext\&pid=S003489102009000300020\&lng =en\&nrm=iso>. Acesso em: 15 jul. 2013.

VERAS, R. P.; DUTRA, S. Perfil do idoso brasileiro: questionário BOAS. Rio de Janeiro: UERJ, 2008. Disponível em: <http://www.crde-unati.uerj.br/liv_pdf/perfil.pdf >. Acesso em: 12 jan. 2013. 